\title{
Síndrome de burnout entre homens de cidade do interior de São Paulo
}

\author{
Burnout syndrome among men in countryside of São Paulo
}

\author{
Síndrome de Burnout en hombres de una ciudad del interior de São Paulo
}

\section{Jorge Luiz Lima da Silva ${ }^{*}$, Felipe dos Santos Costa ${ }^{2}$, Luiz Carlos dos Santos Bento ${ }^{3}$, Giulia Lemos de Almeida $^{4}$, Cristina Portela da Mota ${ }^{5}$, Cláudia Maria Messias ${ }^{6}$.}

Como citar esse artigo. da SIlva, JLL; Bento, LCS; de Almeida, GL; da Mota, CP; Messias, CM. Síndrome de burnout entre homens de cidade do interior de São Paulo. Revista Pró-UniverSUS. 2021 Jan./Jun.; 12 (1): 02-08.

\section{Resumo}

Objetivo: investigar a suspeição de síndrome de burnout entre o público masculino usuário do Serviço Único de Saúde de Bananal. Método: estudo epidemiológico observacional, descritivo seccional. A amostra totalizou 370 participantes. Utilizou-se questionário autoaplicado, abordando hábitos de vida, medidas antropométricas, e escala Maslach Burnout Inventory (MBI). Foi realizada análise descritiva de regressão logística binária. Resultados: identificou-se a prevalência global de 53\%, quando utilizado como critério o desequilíbrio de uma das dimensões da escala. Quando vista a combinação das três dimensões, a prevalência foi de $12,4 \%$; e aqueles que apresentaram despersonificação elevada totalizaram 22,7\%. Mantiveram diferença estatística significativa e risco para a síndrome de burnout as variáveis: possuir companheiro (a); ser provedor da família; e etilismo regular. E como fatores de proteção: tabagismo; consumo de drogas; elevado número de refeições ao dia; e a realização de práticas integrativas e complementares. Conclusão: a prevalência de suspeição reflete a vulnerabilidade da população masculina. A baixa frequência do grupo à rede básica de saúde e o perfil sociocultural masculino são fatores colaborativos para baixa adesão a práticas saudáveis.

Palavras-chave: Esgotamento Profissional; Saúde do Homem; População Rural.

\begin{abstract}
Objective: to investigate the suspicion of burnout syndrome among the male users of the Unified Health Service of Bananal. Method: observational, descriptive, sectional epidemiological study. The sample totaled 370 participants. A self-administered questionnaire was used, addressing life habits, anthropometric measurements, and the Maslach Burnout Inventory (MBI) scale. Descriptive analysis of binary logistic regression was performed. Results: the global prevalence of 53\% was identified, when the imbalance of one of the dimensions of the scale was used as a criterion. When the combination of the three dimensions was seen, the prevalence was $12.4 \%$; and those who showed high depersonification totaled $22.7 \%$. The following variables maintained a statistically significant difference and risk for burnout syndrome: having a partner; be a family provider; and regular drinking. And as protective factors: smoking; drug use; high number of meals a day; and carrying out integrative and complementary practices. Conclusion: the prevalence of suspicion reflects the vulnerability of the male population. The low frequency of the group to the basic health network and the male socio-cultural profile are collaborative factors for low adherence to healthy practices. Y como factores protectores: fumar; el consumo de drogas; alto número de comidas al día; y la realización de prácticas integradoras y complementarias.

Keywords: Burnout, Professional; Men's Health; Rural Population.
\end{abstract}

\section{Resumen}

Objetivo: investigar la sospecha de síndrome de burnout entre los usuarios masculinos del Servicio Unificado de Salud de Bananal. Método: estudio epidemiológico observacional, descriptivo, seccional. La muestra totalizó 370 participantes. Se utilizó un cuestionario autoadministrado que abordaba los hábitos de vida, las mediciones antropométricas y la escala del Inventario de Burnout de Maslach (MBI). Se realizó un análisis descriptivo de regresión logística binaria. Resultados: se identificó la prevalencia global del 53\%, cuando se utilizó como criterio el desequilibrio de una de las dimensiones de la escala. Cuando se observó la combinación de las tres dimensiones, la prevalencia fue del 12,4\%; y aquellos con alta despersificación totalizaron $22.7 \%$. Las siguientes variables mantuvieron una diferencia estadísticamente significativa y el riesgo de síndrome de burnout: tener una pareja; ser un proveedor familiar; y beber regularmente. Conclusión: la prevalencia de sospecha refleja la vulnerabilidad de la población masculina. La baja frecuencia del grupo a la red básica de salud y el perfil sociocultural masculino son factores de colaboración para la baja adherencia a las prácticas saludables.

Palabras clave: agotamiento profesional; salud del hombre; población rural.

Afiliação dos autores:

${ }^{1 *}$ Docente, Programa de Pós-graduação em Saúde Coletiva/Universidade Federal Fluminense/Niterói/Rio de Janeiro/Brasil. E-mail: jorgeluizlima@gmail.com ORCID: http://orcid. org/0000-0002-2370-6343

${ }^{2}$ Enfermeiro. Prefeitura Municipal de Resende/ Secretaria Municipal de Saúde, Resende, RJ, Brasil. Email: felipedosantoscosta@gmail.com ORCID: http://orcid.org/0000-0003 4045-3816

${ }^{3}$ Enfermeiro. Universidade Federal Fluminense, Niterói, RJ, Brasil. Email: luizc.be@gmail.com ORCID: http://orcid.org/0000-0002-5669-9741

${ }^{4}$ Acadêmica de enfermagem. Universidade Federal Fluminense, Niterói, RJ, Brasil. Email: giulialemos@id.uff.br ORCID: http://orcid.org/0000-0003-1783-3298

${ }^{5}$ Discente. Universidade Federal Fluminense, Niterói, RJ, Brasil. E-mail: motacristinap@gmail.com ORCID: http://orcid.org/0000-0001-7496-3385

${ }^{6}$ Doutora e Docente da Universidade Federal Fluminense, Niterói, RJ, Brasil. E-mail: marimessi1512@gmail.com Orcid: https://orcid.org/0000-0002-1323-0214

* Email de correspondencia: jorgeluizlima@gmail.com

Recebido em: 12/03/21. Aceito em: 25/04/21. 


\section{Introdução}

O Sistema Único de Saúde (SUS) se tornou a principal forma de acesso através da Estratégia de Saúde da Família pautada nos princípios da universalidade, equidade, integralidade e na reorganização do sistema de saúde, dando papel central a Atenção Básica ${ }^{1}$.

Pautados nos determinantes sociais da saúde, a atenção básica busca identificar e desenvolver estratégias de saúde próprias a populações vulneráveis, com foco na prevenção e promoção da saúde. Esses são definidos como fatores que influenciam, afetam e/ ou determinam a saúde da população, ou parte dela, e podem ser analisados individualmente em relação ao desfecho, mas somente quando analisados de forma conjunta possibilitam estabelecer o real estado de saúde de um indivíduo ou população ${ }^{2}$.

O ambiente ao qual o indivíduo se insere é um importante determinante social e pode determinar diversos outros fatores. A respeito da população do campo, é possível relacionar o ambiente com a baixa renda, a ausência de saneamento básico, a inadequação das moradias e os baixos índices de escolaridade, apresentando-se como alguns dos desafios enfrentados por essa população. Dentre os agravos que mais acometem a população rural, as relacionadas ao trabalho são as mais evidentes, e podem estar relacionadas com a dificuldade de acesso aos serviços de saúde ${ }^{3}$.

Além do ambiente de inserção, a questão do gênero passa a ser importante determinante. $\mathrm{O}$ público masculino, de maneira geral, não possui o hábito de frequentar as unidades de saúde, salvo em situações extremas. A resistência pela busca dos serviços pode ser explicada, por meio do papel desempenhado por ambos os sexos, ao longo da história da sociedade. O papel do homem sempre foi tido como de provedor da família, e a ideia prevalece mesmo após a inserção da mulher no mercado de trabalho. Tal cultura propagou a máxima de que não podem adoecer, ou demonstrar vulnerabilidade, o que contribuiu para a resistência desse público a frequentar os serviços de saúde 4 .

Recorrentemente, a população masculina, quando busca os serviços de saúde não o faz por meio da atenção básica, se inserindo em níveis mais especializados da rede, devido à procura por atendimento apenas em casos instaurados e avançados na morbidade, o que gera elevação nas despesas do SUS. Ao alterar a forma de inserção dessa população, na rede não só os custos relativos ao processo seriam menores, mas a qualidade de vida seria elevada, por promover a saúde e prevenir danos evitáveis 5 .

Visando alterar esse panorama foi instituída a Política Nacional de Atenção Integral à Saúde do Homem (PNAISH), sendo o Brasil pioneiro dentre os países da América Latina a adotar uma política de saúde direcionada a esse público, contemplando indivíduos com idades entre 20 e 59 anos $^{6}$.

A saúde mental compõe um dos cinco grandes grupos de enfermidades e agravos relacionados de forma relevante ao gênero masculino. Essa se destaca por receber, ao longo das décadas, maior atenção estando relacionada à integralidade dos sujeitos e aos diversos determinantes de saúde, dentre os quais o trabalho surge ${ }^{7}$. Considerado um dos eixos mais importantes da vida humana, a atividade laboral, assume papel vital no processo de subjetivação e de reconhecimento social. $\mathrm{O}$ contexto social e as diversas dinâmicas relacionadas ao ambiente de trabalho são fatores de impacto, no que concerne à saúde do trabalhador, sendo responsáveis por sofrimento psíquico ${ }^{8}$.

A forma como as relações de trabalho se apresenta na atualidade é o principal fator indutor de agravos psíquicos entre os trabalhadores, acometendo cerca de $30 \%$ dessa população. Muitas vezes, a relação de trabalho e seus fatores associados levam os trabalhadores ao seu limite físico e psicológico, acarretando a elevação do número de afastamentos por motivos psicológicos, e por períodos prolongados?.

Devido ao impacto gerado pelo trabalho na saúde criou-se o conceito de Saúde do Trabalhador, pautado nos conceitos de promoção, proteção, recuperação e reabilitação da saúde dessa população, estimulando a propagação da informação, vigilância epidemiológica, capacitação dos profissionais da saúde, pesquisa na área a e educação da população. Os trabalhadores que apresentam certo grau de sofrimento psíquico tendem a desenvolver uma série de problemas associados, dentre os quais a síndrome de burnout (SB), definida como resposta grave aos estressores crônicos relacionados ao ambiente laboral ${ }^{10}$.

O burnout difere de outras formas de reação ao estresse por evolver comportamentos e posturas negativas que interferem diretamente a prática laboral e as relações interpessoais do profissional acometido. Provoca danos práticos e emocionais, tanto para o trabalhador quanto para a instituição, enquanto os quadros de estresse tradicionais estão mais relacionados ao esgotamento pessoal, sendo prejudicial ao indivíduo, mas não afetando, de forma direta, sua relação com o trabalho ${ }^{11}$.

Nesse contexto, surge a pergunta de pesquisa: existe suspeição da síndrome de burnout entre homens do campo, e quais as suas possíveis associações? Assim, o objetivo do estudo é investigar a suspeição de síndrome de burnout entre o público masculino usuário do SUS de Bananal - SP.

\section{Método}

O presente trabalho trata-se de estudo 
epidemiológico observacional, descritivo seccional. Foi realizado com moradores homens do município de Bananal - SP. Segundo o IBGE (2018), conta com uma população estimada de 10.896 pessoas, das quais 5.051 são do sexo masculino ${ }^{12}$. Em relação as características profissionais da população, uma das principais é o trabalho com a terra, normalmente de maneira informal, sendo o último o mais prevalente ${ }^{13}$.

Segundo dados do IBGE (2018), existem cinco estabelecimentos vinculados ao Sistema Único de Saúde (SUS) no município, das quais: quatro pertencem à esfera municipal; e um possui caráter privado $^{12}$. Devido à escassez de serviços particulares de saúde, a população apresenta elevada dependência dos serviços públicos de saúde.

O universo do estudo totalizou 386 sujeitos, onde foram incluídos moradores da cidade de Bananal, do sexo masculino, entre 18 e 65 anos de idade incompletos, trabalhadores com carteira assinada e informais, excluindo indivíduos que se mudaram há menos de seis meses para a cidade, pois se entendeu que, para ser morador, deveriam estar habituados como cidadãos na dinâmica urbana da cidade, e os desempregados a mais de 6 meses. Houve perda total de 4,32\% (16) da amostra, devido aos critérios de inclusão e exclusão, o que totalizou 370 indivíduos na população final, considerando um número de 12 pessoas a mais, contando com eventuais perdas.

O questionário aplicado conteve cerca de 50 variáveis relacionadas aos hábitos de vida, por exemplo, número de refeições por dia, consumo de industrializados, realização de práticas integrativas e complementares (PIC); sociodemográficas (raça/cor, idade), de saúde. Em conjunto com o preenchimento dos questionários foram aferidas medidas antropométricas, sendo: peso; altura; relação cintura/quadril, nas unidades de atendimento, e em visitas domiciliares.

Utilizou-se a escala Maslach Burnout Inventory (MBI) a qual avalia três dimensões: exaustão/ esgotamento emocional (EE), com nove afirmativas; despersonificação (DP), com cinco afirmativas; e realização profissional (RP), com oito afirmativas. Cada afirmativa possuiu escala de frequência variando escores de acima de quarenta até menores que seis, seguindo os pontos de cortes descritos na tabela $1^{14}$.

Utilizou-se o Alpha de Cronbach para avaliar a confiabilidade interna da escala, sendo a geral 0,843 . Avaliou-se ainda a confiabilidade interna das dimensões de forma 4individual, para o esgotamento emocional o resultado foi 0,918, para despersonificação 0,778 e para realização profissional 0,743. Levando em consideração as informações anteriores, o instrumento, demonstrou confiabilidade interna satisfatória para análise da suspeição de burnout.
Tabela 1. Pontos de corte das dimensões da síndrome de burnout.

\begin{tabular}{cccc}
\hline DIMENSÕES & Alto & Médio & Baixo \\
\hline Esgotamento emocional & $\geq 27$ & $19-26$ & $<19$ \\
Despersonificação & $\geq 10$ & $6-9$ & $<6$ \\
Realização profissional & $\leq 33$ & $34-39$ & $\geq 40$ \\
\hline
\end{tabular}

Fonte: Benevides-Pereira ${ }^{14}, 2002$.

Foram realizadas análises descritivas (uni, bi e multivariada), observados os resultados dos testes de qui-quadrado (x2). Foram consideradas como potenciais confundidoras as covariáveis que apresentaram significância estatística, foi considerado o valor de $\mathrm{p}$ $\leq 0,05$. Utilizou-se o programa Statistical Package for the Social Sciences versão 21(SPSS $\left.{ }^{\circledR}\right)$ para o processo de análise.

Este estudo foi aprovado pelo Comitê de Ética e Pesquisa da Faculdade de Medicina do Hospital Universitário Antônio Pedro, no ano de 2018, e seguiu o preconizado pela Resolução $\mathrm{n}^{\circ} 466 / 12$, sobre pesquisas com seres humanos. Parecer número 2.617.228. A coleta de dados ocorreu no primeiro semestre de 2018, e faz parte de dissertação de mestrado em Saúde Coletiva da Universidade Federal Fluminense.

\section{Resultados}

Identificou-se a prevalência global foi de 53\%, quando utilizado como critério o em desequilíbrio de uma das dimensões da escala. Quando vista a combinação das três dimensões, a prevalência foi de $12,4 \%$; e, por fim, aqueles que apresentaram despersonificação elevada totalizaram 22,7\%. Valendo-se dos pontos de corte admitidos para cada uma das dimensões, chegou-se ao resultado de 72 (19,45\%) entrevistados apresentaram alto esgotamento emocional ( $\geq 27$ pontos); 157 (42,43\%) alta despersonificação ( $\geq 10$ pontos); e 27 (7,29\%) baixa realização profissional ( $\geq 40$ pontos) (tabela 2 ).

Após análise dos dados obteve-se o perfil sociodemográfico da amostra. Quanto à cor da pele/ raça, $193(52,2 \%)$ entrevistados se autorreferiram pardos; em relação à idade $172(46,5 \%)$ dos indivíduos

Tabela 2. Suspeição de burnout entre homens de cidade no interior do Estado de São Paulo - SP, estratificada de acordo com as dimensões / $\mathrm{N}=370$.

\begin{tabular}{cccc}
\hline DIMENSÕES & Alto n (\%) & Médio n (\%) & Baixo n (\%) \\
\hline Esgotamento emocional & $072(19,45 \%)$ & $044(11,89 \%)$ & $254(68,64 \%)$ \\
Despersonificação & $157(42,43 \%)$ & $064(17,29 \%)$ & $149(40,27 \%)$ \\
Realização profissional & $297(80,27 \%)$ & $046(12,43 \%)$ & $027(07,29 \%)$ \\
\hline
\end{tabular}

Fonte: Autores, 2021. 
encontravam-se acima de 37 anos. Em relação à situação conjugal, 220 (59,5\%) indivíduos afirmaram não possuir companheira; dentre os entrevistados 200 $(54,1 \%)$ relataram não possuir filhos.

A renda familiar média identificada foi entre dois e três salários-mínimos, nessa faixa encontraram-se 132 $(35,7 \%)$ dos entrevistados. A maioria dos entrevistados, $177(47,8 \%)$ possuía escolaridade "ensino médio completo"; dentre os entrevistados 259 (70\%) exerciam atividade laboral com carteira assinada. Afirmaram ser provedores de suas famílias 179 entrevistados (48,4\%).

No grupo estudado, $84(22,7 \%)$ dos participantes referiram ser tabagistas; $188(50,8 \%)$ relataram ser etilistas; e $57(15,4 \%)$ informaram fazer uso regular de outras drogas, não especificadas; 244 (65,9\%) declararam ser sedentários. Quanto aos hábitos alimentares, 196 (53,0\%) indivíduos informaram fazer mais de três refeições ao dia e "não consumirem frutas todos os dias", com 132 (35,7\%); enquanto o consumo de alimentos ultraprocessados "uma a três vezes por semana", 117 (31,6\%). Dos participantes, 312 (84,3\%) afirmavam não ter buscado por PIC; aqueles que buscaram alguma prática referiram a utilização de chás, $54(14,6 \%)$.

Dentre os homens entrevistados 267 (72,2\%) relataram ter ido ao menos uma vez à unidade de saúde do município, dos quais 127 (34,3\%) afirmaram ir às unidades uma vez ao ano. Dentre os participantes, $230(62,2 \%)$ referiram frequentar os serviços de saúde trimestralmente. Os principais motivos citados foram: consulta de rotina, referida por 81 (21,9\%); imunização, referida por 58 (15,7\%); e algum tipo de queixa álgica, referido por 48 (13,0\%). Disseram não realizar acompanhamentos no SUS 301 entrevistados (81,4\%).

A tabela 3 apresenta variáveis significativas: como indivíduos entre 30 e 41 anos; faixa de renda mais elevada, provedor da família; praticar atividade física; e não ser usuário de tabaco ou drogas; e realizar poucas refeições diárias (até 3).

De acordo com o modelo de regressão utilizado, e após ajustes pelos potenciais variáveis de confundimento, mantiveram diferença estatística significativa e risco as seguintes variáveis: situação conjugal; provedor da família; etilista regular; tabagismo; consumo de drogas; número de refeições; realização de PIC.

Ser provedor da família apresentou risco aproximadamente dez vezes maior para o desfecho, sendo a variável com maior impacto, seguida por consumo regular de bebidas alcoólicas e possuir companheira (o). Contudo, aqueles participantes que afirmaram consumir drogas, realizar PIC, e se alimentar bem (mais de três refeições diárias), a análise indicou proteção.
Tabela 3. Prevalência de suspeição de burnout entre homens, de cidade no interior do Estado de São Paulo - SP/ N=370.

\begin{tabular}{|c|c|c|c|c|}
\hline VARIÁVEIS & TN & $\mathrm{n}$ & $\%$ & $\underset{n}{\text { Valor de }}$ \\
\hline Idade & & & $\mathrm{DP} \pm 14,53$ & 0,007 \\
\hline Até 30 anos & 160 & 072 & 45,00 & \\
\hline 30- 41 anos & 122 & 080 & 65,57 & \\
\hline $41-59$ anos & 065 & 033 & 50,77 & \\
\hline Acima de 60 anos & 023 & 011 & 47,83 & \\
\hline Situação conjugal & & & & 0,043 \\
\hline Com companheiro (a) & 150 & 089 & 59,33 & \\
\hline Sem companheiro (a) & 220 & 107 & 48,64 & \\
\hline Faixa de renda & & & & 0,006 \\
\hline Até $2 \mathrm{SM}$ & 191 & 088 & 46,07 & \\
\hline Acima de $2 \mathrm{SM}$ & 179 & 108 & 60,34 & \\
\hline Provedor da familia & & & & $<0,0001$ \\
\hline É provedor & 179 & 131 & 73,18 & \\
\hline Não é provedor & 191 & 065 & 34,03 & \\
\hline $\begin{array}{l}\text { Atividade fisica } \\
\text { Sim }\end{array}$ & 126 & 080 & 63,49 & 0,004 \\
\hline Não & 244 & 116 & 47,54 & \\
\hline Etilista regular & & & & 0,050 \\
\hline Sim & 188 & 109 & $\mathbf{5 7 , 9 8}$ & \\
\hline Não & 182 & 087 & 47,80 & \\
\hline Tabagismo & & & & 0,009 \\
\hline Sim & 084 & 034 & 40,48 & \\
\hline Não & 286 & 162 & 56,64 & \\
\hline Consumo de drogas & & & & $<0,0001$ \\
\hline Sim & 057 & 018 & 31,58 & \\
\hline Não & 313 & 178 & 56,87 & \\
\hline Número de refeições & & & & $<0,0001$ \\
\hline Até 3 refeições por dia & 174 & 109 & 62,64 & \\
\hline Mais que 3 refeições por dia & 196 & 087 & 44,39 & \\
\hline Realização de PIC & & & & 0,012 \\
\hline Sim & 058 & 022 & 37,93 & \\
\hline Não & 312 & 174 & 55,77 & \\
\hline
\end{tabular}

Legenda: $\mathrm{TN}=$ total no estrato. $\mathrm{n}=$ número de homens suspeitos. $\%=$ prevalência $\mathrm{SM}=$ salários mínimos.PIC $=$ Práticas Integrativas e Complementares. $\mathrm{P}=$ Teste do qui-quadrado de Pearson.

Fonte: Autores, 2021.

\section{Discussão}

Os estudos relacionados à $\mathrm{SB}$ apresentam populações com características diferentes à deste estudo. Grande parte dos trabalhos é referente a grupos profissionais, majoritariamente a enfermagem e à docência, e composta por populações de gênero misto ${ }^{15}$. Tais informações não impossibilitam a comparação dos achados deste estudo com demais pesquisas, porém, dificultam o enquadramento em valores de referência, tendo em vista a disparidade entre populações.

Identificou-se a prevalência global de $53 \%$ de acometimento da população, correspondente a 196 indivíduos. Estudo realizado com trabalhadores do setor hidroviário, $\operatorname{com} 83,5 \%$ da amostra sendo do sexo masculino, apresentou prevalência global de 78,1\% ${ }^{16}$, evidenciando como os resultados estiveram próximos.

A influência da situação conjugal sobre o desfecho não é unânime, pois, mesmo que a maioria dos estudos aponte para associação da síndrome ao fato de ser solteiro ${ }^{17}$, há alguns que apontam para a maior prevalência entre o grupo que possui companheira (o), como esta pesquisa. Dentre os que afirmam viver em 
Tabela 4. Estimativas do modelo selecionado, na análise de regressão logística múltipla binária, entre homens, de cidade no interior do Estado de São Paulo - SP/ N=370.

\begin{tabular}{lccc}
\hline \multicolumn{1}{c}{ VARIÁVEIS } & Valor de & RP & IC 95\% \\
\hline Situação conjugal & 0,043 & 1,10 & $1,053-3,658$ \\
Provedor da família & 0,000 & 9,50 & $6,431-23,452$ \\
Etilista regular & 0,050 & 1,40 & $1,349-3,890$ \\
Tabagismo & 0,009 & 0,59 & $0,228-0,782$ \\
Consumo de drogas & 0,000 & 0,59 & $0,207-0,863$ \\
Número de refeiç̃̃es & 0,000 & 0,56 & $0,237-0,661$ \\
Realização de PIC & 0,012 & 0,61 & $0,217-0,889$ \\
\hline
\end{tabular}

Legenda: $\mathrm{P}=$ Teste do qui-quadrado de Pearson. $\mathrm{RP}=$ Razão de prevalência. IC $95 \%=$ Intervalo de Confiança de $95 \%$.

Fonte: Autores, 2021

companhia de alguém, 59,33\% apresentaram suspeição para a síndrome. Essa associação pode estar relacionada a dificuldades interpessoais e crises de relacionamento, tendo em vista que ser compromissado não significa ter apoio emocional.

Constatou-se, ainda, associação entre a variável ser provedor da família, 73,18\%. Não foi possível identificar na literatura associação semelhante entre esta variável e o desfecho, entretanto, atribui-se a esse achado fatores relacionados com a variável possuir filhos, pois há pressão psicossocial sobre o homem, sendo responsável pela manutenção de outra vida e cobrado socialmente ${ }^{18}$.

O consumo regular de álcool apresentou, aproximadamente, uma vez e meia mais risco para o desfecho na população estudada. O etilismo pode estar associado ao estresse advindo do ambiente de trabalho, uma vez que, a manifestação comportamental promove efeitos ansiolíticos que levam ao relaxamento e tranquilidade. ${ }^{16}$ Estudo realizado com médicos militares em hospital no Rio de Janeiro, apontou a relação entre a SB e o etilismo, a prevalência do consumo de álcool dentre os acometidos pela síndrome foi de 28,6\%, corroborando os achados deste estudo ${ }^{19}$.

A prevalência de tabagismo deu $22,7 \%$, em consonância com a média mundial de 20,7\%; mas, elevada, quando comparada à média nacional $(14,7 \%)$ ${ }^{20}$. O tabagismo e estresse apresentam importante relação de risco, pois o ato de fumar é considerado uma forma de relaxar, como relatado por tabagistas. Nesta pesquisa foi possível identificar relação entre o grupo não tabagista e a síndrome. Embora estudos afirmem que o tabagismo é fator de risco para o estresse, e consequentemente para burnout, estudo realizado com equipe de enfermagem de hospital em São Paulo apontou para a maior incidência da síndrome entre os não fumantes, com $51,2 \%$, valor próximo ao encontrado neste estudo, 56,64\% ${ }^{15}$.

Foi observada a associação do não consumo de drogas com o desfecho. Embora, não se pode afirmar que o uso de drogas seja determinante de proteção para a síndrome. A sobrecarga de trabalho e o estresse, quando associados a substâncias lícitas, podem favorecer o consumo de outras drogas, dinâmica que aliada ao trabalho insatisfatório, e maior exposição a riscos ocupacionais, podem elevar a possiblidade dos indivíduos recorrerem ao consumo abusivo ${ }^{21}$. Deve-se considerar também os efeitos deletérios relacionados ao consumo dessas substâncias, como prejuízos à saúde física, social e psíquica, além da redução do rendimento profissional, bem como alterações no raciocínio que elevam a suscetibilidade a acidentes ${ }^{21}$.

Identificou-se relação da síndrome com os participantes que consumiam menos de três refeições ao dia, em consonância ao observado em pesquisa, onde a maioria dos participantes relataram comer menos $^{22}$. Períodos de estresse crônico podem alterar o comportamento alimentar, como no caso de burnout. No entanto, o padrão mais comum é o aumento do apetite como mecanismo de compensação a situações estressoras, como exposto em estudo realizado na Finlândia, onde evidenciou-se a relação da síndrome com a alteração dos hábitos alimentares ${ }^{23}$. Ou seja, comer em excesso pode ser de certa forma um fator confortante/ amenizador.

Dentre os indivíduos que não praticavam nenhum tipo de PIC a prevalência para a suspeição foi maior, quando comparada ao grupo que realizava, porém não foi possível encontrar na literatura estudo sobre essa associação. Mas, sabe-se que essa prática promove a redução dos níveis de estresse, assim como o desenvolvimento da habilidade de enfrentamento ${ }^{24}$. Entendendo o estresse como precursor de burnout, é possível afirmar que o manejo adequado reduziria, substancialmente o risco.

As PICs podem ser definidas como conjunto de práticas terapêuticas que buscam a promoção e recuperação da saúde utilizando-se de meios naturais ${ }^{24}$. Atualmente, são reconhecidas vinte nove modalidades ${ }^{24}$, dentre as quais, três são as mais relatadas pelos entrevistados: a musicoterapia, o uso de plantas medicinais/fitoterapia, e a meditação.

Percebem-se inúmeras tentativas de explicar a pouca procura do público masculino ao serviço de saúde, como por exemplo, características históricas e psicossocioculturais da masculinidade e a estrutura dos serviços, em grande parte velados a população feminina e infantil. Nesse contexto, a ausência ou a invisibilidade dos homens sinaliza a inadequação entre as necessidades e/ou expectativas desses atores ${ }^{25}$.

Nessa lógica, a saúde mental é produto de interações complexas, que incluem fatores biológicos, psicológicos e sociais. Entende-se que os determinantes sociais de saúde estão diretamente associados na maneira como se dá o desenvolvimento dos transtornos mentais, os quais podem influenciar a saúde física e mental dos indivíduos ${ }^{26}$.

O debate a respeito desse assunto é urgente. Ao investigar-se essa questão, sobretudo em estratos populacionais mais desfavorecidos, percebe-se que as 
situações de vulnerabilidade, a escassez dos recursos, os eventos produtores de estresse como a falta de apoio social, a falta de trabalho e perspectivas de futuro, pouca escolaridade, menor número de bens, condições precárias de moradia e baixa renda são desencadeadores de sofrimento ${ }^{26}$.

Ao avaliar-se a saúde mental, sobretudo das coletividades, faz-se relevante os transtornos mentais comuns (TMC). Trata-se de tipo de transtorno em que sua conceituação transcende categorias diagnósticas fixas estabelecidas, como a CID-10. É a nomenclatura que se aplica àqueles indivíduos que apresentam sintomas psíquicos inespecíficos e cujos diagnósticos não estão compreendidos em uma única categoria, dentro da psiquiatria ${ }^{27}$.

A prevalência desse tipo de transtorno entre os usuários da atenção básica é elevada, especialmente em países como o Brasil ${ }^{28}$. Estudos de revisão sistemática brasileiros apontam variação entre $29,6 \%$ a $64,3 \%$ da prevalência dos $\mathrm{TMC}^{29}$. Majoritariamente, esses grupos envolvem mulheres, trabalhadores, adolescentes, gestantes e profissionais de saúde, o que desperta o interesse para que mais investigações sejam dirigidas entre grupos diferentes, como homens. Dessa forma, pode-se precocemente identificar sinais iniciais de danos à saúde mental do grupo.

\section{Limitações do estudo}

A principal limitação deste estudo é referente ao desenhado utilizado, pois estudos seccionais se restringem a observação da população em um determinado ponto temporal, onde exposição e desfecho são analisados simultaneamente, o que dificulta a delimitação da causa e efeito, não havendo como estabelecer com segurança a sequência temporal dos eventos ${ }^{30}$.

As variáveis de confundimento também se enquadram como fator de limitação do estudo, entretanto, como relatado anteriormente, a escassez de estudos sobre burnout com amostra populacional próxima a deste estudo dificultou a identificação de variáveis descritas anteriormente na literatura. Dessa forma, se constituiu desafio identificar variáveis de confundimento que pudessem ser comparadas com os achados, embora se tenha aplicado os potenciais variáveis dessa natureza no modelo de regressão. ${ }^{30}$

\section{Conclusão}

A prevalência global da suspeição da síndrome na amostra estuda foi de 53\%, em consonância com outros estudos. As características observadas refletem o perfil do público masculino em relação à reduzida prática do autocuidado, adoção de hábitos de risco e baixa adesão aos serviços de saúde, em nível de prevenção e promoção de saúde.

Levando em consideração o contexto da rede de saúde presente no município, onde o atendimento é realizado praticamente de forma exclusiva pela rede básica do SUS, cabe aos entes responsáveis traçar estratégias de aproximação desse público às unidades de saúde, proporcionando a execução de ações educativas junto à população e a capacitação de profissionais para atender as especificidades da saúde do homem.

Diante dos achados, fica explícita a necessidade de desenvolvimento de novos estudos voltados para a população masculina, do campo, de saúde mental e a interrelação desses aspectos com a síndrome

\section{Referências}

1. Feltrin AFS, Coneglian TV. A nova política nacional de atenção básica e o contexto atual de saúde: uma reflexão crítica. CuidArte: Enfermagem, 2019;13(1):56-61. Disponível em: <http://www.webfipa.net/facfipa/ner/ sumarios/cuidarte/2019v1/56.pdf>.

2. Figueiredo DS, Heidemann ITSB, Fernandes GCM, Arawaka AM, Oliveira LS, Magagnin AB. Promoção da saúde articulada aos determinantes sociais: possibilidade para a equidade. Revista de Enfermagem UFPE, on-line, 2019;13(4):943-951. doi: http://doi.org/10.5205/1981-8963v13i4a239123p943-951-2019.

3. Ruckert B, Aranha AVS. Lutar por saúde é lutar por reforma agrária: estudo sobre práticas de saúde no Movimento dos Trabalhadores Rurais Sem Terra. Saúde e Sociedade, 2018;27(1):116-127. doi: http://dx.doi. org/10.1590/s0104-12902018170158.

4. Assis NO, Rodrigues J, Christóforo BEB, Tacsi YRC. Atuação dos enfermeiros frente à política nacional de atenção integral a saúde do homem: um estudo exploratório. Arquivo de Ciências da Saúde UNIPAR, 2018;22(3):151-156.doi: https://doi.org/10.25110/arqsaude. v22i3.2018.6397

5. Abreu TCA, Oliveira GS, FeitosaANA, Silva ML, Medeiros RLSFM Atenção integral à saúde do homem: adesão da polícia militar. Revista de Enfermagem da UFPE on-line, 2018;12(10):2635-2642. doi: https://doi. org/10.5205/1981-8963-v12i10a237503p2635-2642-2018.

6. Pereira J, Klein C, Meyer DE. PNAISH: uma análise de sua dimensão educacional na perspectiva de gênero. Saúde e Sociedade,2019;28(2):132146. doi: http://dx.doi.org/10.1590/s0104-12902019170836.

7. Ribeiro MC, Barros AC, Correia MS, Lessa RO, Tavares LN, Chaves JB. Atenção psicossocial e satisfação no trabalho: processos dialéticos na saúde mental. RIES, 2018;7(1):55-67. doi: https://doi.org/10.33362/ries. v7i1.1102.

8. Amazarray MR, Oliveira GF, Feijo FR. Contexto de Trabalho e Transtornos Mentais Comuns em Trabalhadores do Judiciário Federal no Rio Grande do Sul, Brasil. Revista Psicologia: Organizações e Trabalho, 2019;19(3):687-694. doi: http://dx.doi.org/10.17652/rpot/2019.3.16744.

9. Melo CF, Cavalcante AKS, Facanha KQ. Invisibilização do adoecimento psíquico do trabalhador: limites da integralidade na rede de atenção à saúde. Trabalho Educação e Saúde, 2019;17(2):e0020132. doi: http://dx.doi.org/10.1590/1981-7746-sol00201.

10. Esteves GGL, Leao AAM, Alves EO. Fadiga e Estresse como preditores do Burnout em Profissionais da Saúde. Revista Psicologia: Organização e Trabalho, 2019;19(3):695-702. doi: http://dx.doi.org/10.17652/ $\mathrm{rpot} / 2019.3 .16943$.

11. Miguez VA, Braga JRM. Estresse, Síndrome de Burnout e suas implicações na saúde docente. Revista Thema, 2018;15(2)704-716. doi: http://dx.doi.org/10.15536/thema.15.2018.704-716.861.

12. Instituto Brasileiro de Geografia e Estatística. Dados estatísticos de 
2018. Brasilia (DF); 2018. Disponível em: <https://www.ibge.gov.br/pt/ inicio.html>.

13. Estado de São Paulo. A cidade: prefeitura municipal de Bananal. Disponível em: $<$ https://www.bananal.sp.gov.br/cidade/index/>.

14. Benevides-Pereira AMT. Burnout: quando o trabalho ameaça o bemestar do trabalhador. São Paulo: Casa do Psicólogo; 2002.

15. Fernandes LS, Nitsche MJT, Godoy I. Associação entre Síndrome de burnout, uso prejudicial de álcool e tabagismo na Enfermagem nas UTIs de um hospital universitário. Ciência e Saúde coletiva, 2018;23(1):203-214. doi: http://dx.doi.org/10.1590/1413-81232018231.05612015.

16. Silva JLL, Souza AC, Soares RS, Teixeira ER, Costa FS, Oliveira MBDB. Prevalência da síndrome de burnout entre trabalhadores hidroviários. Rev enferm UERJ, 2018; 26:e26131. doi: http://dx.doi.org/10.12957/ reuerj.2018.26131.

17. Silva E, Dias P, Rodrigues A. Satisfação laboral e burnout em assistentes gerontológicos. Psic., Saúde \& Doenças, 2019; 20(3): 788-802. doi: http://dx.doi.org/10.15309/19psd200318.

18. Trindade LL, Lautert L. Síndrome de Burnout entre os trabalhadores da Estratégia de Saúde da Família. Revista da Escola de Enfermagem da USP, 2010;44(2):274-279. doi: http://dx.doi.org/10.1590/S008062342010000200005 .

19. Lima CRC, Sepúlveda JLM, Lopes PHTNP, Fajardo HSR, Sousa MM, Ferreira Júnior MC et al. Prevalência da síndrome de burnout em médicos militares de um hospital público no Rio de Janeiro. Revista Brasileira de Medicina do Trabalho, 2018;16(3):287-296. doi: http://dx.doi.org/10.5327/ Z1679443520180297.

20. Oliveira RM, Santos JLF, Furegato ARF. Prevalência e perfil de fumantes: comparações na população psiquiátrica e na população geral. Revista Latino-Americana de Enfermagem, 2019;27:e. 3149. doi: http:// dx.doi.org/10.1590/1518-8345.2976.3149.

21. Scholze AR, Martins JT, Galdino MJQ, Ribeiro RP. Ambiente ocupacional e o consumo de substâncias psicoativas entre enfermeiros. Acta Paulista de Enfermagem, 2017;30(4):404-411, ago. 2017. doi: http://dx.doi. org/10.1590/1982-0194201700060.

22. Costa FD, Teo CRPA, Almeida JS. Vulnerabilidade ao estresse e alimentação: um estudo no contexto do trabalho. Ciência Médica, 2015;25(2). doi: http://dx.doi.org/10.15448/1980-6108.2015.2.20372.

23. Nevanperã NJ, Hopsu L, Kuosma E, Ukkola O, Uitti J, Laitinen $\mathrm{JH}$. Burnout ocupacional, comportamento alimentar e peso entre mulheres trabalhadoras. The American Journal of Clinical Nutrition, 2012;95(4):934 -943. doi: https://doi.org/10.3945/ajcn.111.014191.

24. Mendes DS, Moraes FS, Lima GO, Silva PR, Cunha TA, Crossetti MGO et al. Benefícios das práticas integrativas e complementares no cuidado de enfermagem. Journal Health NPEPS, 2019;4(1):302-318. doi: http://dx.doi.org/10.30681/252610103452.

25. Sousa AR, Queiroz AM, Florencio RMS, Portela PP, Fernandes JD, Pereira Á. Homens nos serviços de atenção básica à saúde: repercussões da construção social das masculinidades. Revista Baiana de Enfermagem, 2016;30(3):1-10. doi: 10.18471/rbe.v30i3.16054.

26. Dimenstein M, Siqueira K, Macedo JP, Leite J, Dantas C. Determinação social da saúde mental: contribuições à psicologia no cuidado territorial. Arquivos Brasileiros de Psicologia. 2017; 69(2): 72-87. Disponível em: $<$ http://pepsic.bvsalud.org/pdf/arbp/v69n2/06.pdf $>$.

27. Lopes CS, Abreu GA, Santos DF, Menezes PR, Carvalho KMB, Cunha CF et al. ERICA: prevalence of common mental disorders in Brazilian adolescents. Revista de Saúde Pública, 2016; 50. doi: https://doi.org/10.1590/ S01518-8787.2016050006690.

28. Souza LS, Barbosa B, Silva CO, Souza A, Ferreira T, Siqueira L. Prevalência de transtornos mentais comuns em adultos no contexto da atenção primária à saúde. Revista Portuguesa de Enfermagem de Saúde Mental, 2017; (18):59-66. doi: http://dx.doi.org/10.19131/rpesm.0193.

29. Menezes ALA, Correia CRM. Clínica da saúde mental na atenção primária em saúde. Módulo 4 do curso de especialização em saúde mental Unasus. Brasília (DF); 2017. Disponível em: <http://repocursos.unasus. ufma.br/especializacao saude mental/repositorio/mod4 pdf.pdf $>$.
30. Fávero LP. Análise de dados: modelos de regressão. Rio de Janeiro: Ed. Campos; 2015. 